\title{
ORIGINAL
}

\section{VALIDACIÓN DE UN CUESTIONARIO DE FRECUENCIA DE CONSUMO DE ALIMENTOS ADAPTADO PARA EL ESTUDIO Y SEGUIMIENTO DE LA POBLACIÓN ADULTA DE LAS ISLAS CANARIAS (*)}

\author{
Armando Aguirre-Jaime (1), Antonio Cabrera de León (1,2,3), Santiago Domínguez Coello (1), \\ Carlos Borges Álamo (1), Lourdes Carrillo Fernández (1), J. Carlos Gavilán Batista (4), María \\ del Cristo Rodríguez Pérez (1), Delia Almeida González (1) \\ (1) Unidad de Investigación Hospital Universitario NS Candelaria y Gerencia de Atención Primaria, Tenerife. \\ (2) Departamento de Medicina Preventiva y Salud Pública, Universidad de La Laguna. \\ (3) Hospital San Juan de Dios, Tenerife. \\ (4) Centro de Salud de El Pinar, El Hierro.
}

\section{RESUMEN}

Fundamento: Registrar los hábitos alimenticios exige de un método válido y fiable. El objetivo del estudio es validar el cuestionario de frecuencia de consumo de alimentos CDCFFQ, que es una adaptación de otro cuestionario, para estudiar la alimentación de la población adulta de Canarias.

Métodos: El cuestionario CDC-FFQ fue administrado a 1.067 personas de la población general (PG) y a 106 estudiantes universitarios (EU), de 19 a 30 años. El segundo grupo fue encuestado también sobre tres recordatorios de 24 horas. Se comparan los nutrientes según CDC-FFQ en PG y EU. Se estiman las correlaciones entre CDC-FFQ y los recordatorios para nutrientes y grupos de alimentos, y la concordancia de consumos de nutrientes y grupos de alimentos en los quintiles extremos, para los estudiantes universitarios.

Resultados: Los valores medios entre nutrientes del CDC-FFQ entre PG, EU y población general con estudios universitarios no mostraron diferencias significativas excepto para la vitamina $\mathrm{B} 12(\mathrm{p}=0.004)$ y la vitamina $\mathrm{D}(\mathrm{p}=0,005)$. Entre el CDC-FFQ y la media de los tres recordatorios se obtuvieron correlaciones en el rango de 0,202-0,601 entre nutrientes ajustados por calorías consumidas para los estudiantes universitarios. Por grupos de alimentos las correlaciones oscilaron para CDC-FFQ y los recordatorios entre 0,2430,542 . La concordancia de nutrientes osciló entre $39 \%$ y $100 \%$ y para grupos de alimentos entre $41 \%$ y $100 \%$.

Conclusiones: El cuestionario $\mathrm{CDC}-\mathrm{FFQ}$ resulta válido para clasificar a los sujetos en los rangos relativos de su nivel de ingesta de alimentos y nutrientes, por lo que podría ser útil en estudios epidemiológicos con valoración de dieta en población canaria adulta.

Palabras clave: Estudios de validación. Alimentos. Nutrición. Evaluación nutricional. Encuestas de nutrición. España.

Correspondencia:

Antonio Cabrera de León

Hospital San Juan de Dios, Ctra. Santa Cruz-La Laguna, 53 38009 Santa Cruz de Tenerife. Canary Islands, Spain Correo electrónico: acabrera@sjd.es

\section{ABSTRACT}

\section{Validation of a Food Intake Frequency Questionnaire Adapted for the Study and Monitoring of the Adult Population of the Canary Islands, Spain}

Background: The registering of eating habits requires a valid and reliable method. The purpose of this study is to validate the food intake frequency questionnaire, CDC-FFQ, which is an adaptation of another questionnaire, in order to assess the nutrition of the adult population of the Canary Islands.

Methods: The CDC-FFQ questionnaire was given to 1,067 individuals taken from the general population (GP) and to 106 university students, aged 19 to 30 . The second group was surveyed also in three 24-hour follow-ups. The nutrients were compared according to the CDC-FFQ in the GP and university students. The correlations were estimated between the CDCFFQ and the follow-ups for nutrients and groups of foods and the concordance of the intakes of nutrients and groups of foods in the extreme quintiles, for the university students.

Results: The mean values between nutrients of the CDCFFQ between the GP, university students and the general population with university studies showed no significant differences except for vitamin B12 ( $\mathrm{p}=0.004)$ and vitamin $\mathrm{D}$ $(p=0.005)$. Correlations between the CDC-FFQ and the mean of the three follow-ups were obtained in the 0.202-0.601 range between nutrients adjusted by calories consumed in the case of the university students. By groups of foods, the correlations ranged between $0.243-0.542$ for the CDC-FFQ and the followups. The concordance of nutrients ranged between $39 \%$ and $100 \%$ and for groups of foods, between $41 \%$ and $100 \%$.

Conclusions: The CDC-FFQ questionnaire is valid for classifying the subjects in the relative ranges of their level of intake of foods and nutrients and, therefore, it could be useful in epidemiological studies with a diet assessment in the adult population of the Canary Islands.

Keywords: Validation studies. Food. Nutrition. Nutrition Assessment. Nutrition Surveys. Spain.

(*) Este estudio ha sido financiado por el FIS (99/0361, 02/1158, 02/1189) y la FUNCIS (45/98). 


\section{INTRODUCCIÓN}

La exposición de las poblaciones a algunos factores de difícil medición, como la alimentación, para valorar su posible asociación con enfermedades como la diabetes, el cáncer, y la aterosclerosis, requiere del empleo de cuestionarios cuya validez y fiabilidad debe estar bien establecida ${ }^{1}$. En estudios que incluyen la alimentación entre los factores considerados, los cuestionarios de frecuencia de consumo de alimentos (FFQ según sus siglas en inglés) son ampliamente utilizados por razones de eficiencia $^{2-4}$.

En España se han creado cuestionarios dirigidos a estimar el consumo de alimentos específicos de la población española como factor asociado al padecimiento de ciertas enfermedades, como el de Benito E y cols para cáncer de cólon ${ }^{5}$, el de Riboli E y cols para cáncer de vesícula validado para población general española ${ }^{6} \mathrm{o}$ el de Martín-Moreno JM y cols para el estudio del cáncer de mama y colon validado en mujeres de toda España ${ }^{7}$. Sin embargo, la población canaria posee una dieta que difiere de la del resto de la población española ${ }^{8}$ al incluir alimentos autóctonos heredados de la población aborigen y otros procedentes de la emigración a Centroamérica, así como al excluir otros alimentos usuales que se consumen poco en Canarias, por lo que el empleo de dichos cuestionarios para estudios en estas islas podría no reflejar la especificidad de su dieta.

Por ello, para analizar el espectro de alimentos de la población canaria hemos adaptado el cuestionario FFQ desarrollado por Martín-Moreno y cols. validado en población española. El cuestionario resultante se denominó "CDC-FFQ" porque su primera aplicación se efectuó en la cohorte del estudio "CDC de Canarias"9. El objetivo del presente estudio es determinar la validez del CDC-FFQ en la estima- ción de los alimentos y nutrientes consumidos por la población de las Islas Canarias.

\section{SUJETOS Y MÉTODOS}

El cuestionario CDC-FFQ se halla disponible en www.icic.es/cuestionarioCDC/docs/encuesta_CDC.pdf. Este cuestionario, a diferencia del de Martín Moreno y $\operatorname{cols}^{7}$, recoge el consumo de 138 alimentos por medio de 125 preguntas cerradas, más 7 preguntas abiertas que añaden unas 13 comidas más. Las preguntas acerca de la cantidad y frecuencia de los alimentos se contestan a través de categorías continuas que ofrecen 11 posibilidades de elección y 5 tamaños de ración distintos. Para precisar el tamaño de las raciones se utilizó un álbum fotográfico a escala natural que muestra diferentes platos y que se le enseña al participante para que pueda comparar la ración que habitualmente come con la que aparece en la fotografía, tomando como la unidad de medida dichas raciones. Para una mejor estimación de la ingesta de frutas y verduras su consumo se explora tanto de forma agrupada como individual. Todas las preguntas se refieren al año anterior a la entrevista. Para conseguir una mejor aproximación en las unidades de medida teniendo en cuenta la cultura canaria, se realizó previamente un estudio piloto donde se observó que el consumo de cereales, gofio, frutos secos y otros alimentos se cuantificaba mejor como "número de cucharones" que generalmente se consumen, ya que se comprobó que el error de medición resultaba inferior a la utilización de unidades alternativas como "número de cucharas", "número de puñados" o "número de racimos", o similares. De esta manera, en el pilotaje de este estudio se comprobó que un cucharón de gofio correspondía a 80 gr, de cereales $50 \mathrm{gr}$, de frutos secos $120 \mathrm{gr}$, de pasas 120 gr, de uvas 130 gr, de legum- 
bres secas $120 \mathrm{gr}$, y de potaje o de comida preparada tipo fabada 60 gr de legumbres y 60 de agua.

Para obtener la cantidad promedio de ingesta diaria de un alimento se convirtió la frecuencia de consumo a su equivalente en unidades de 24 horas. A continuación, se multiplicó por la cantidad de alimento de cada ración expresada en gramos y se obtuvo la cantidad diaria de gramos consumidos. A la cantidad diaria de gramos consumidos se le aplicó la Tabla de Composición de Alimentos de Mataix Verdú ${ }^{10}$, ajustando según porción comestible y peso perdido al cocinar y se convirtió en sus respectivos nutrientes. La ingesta diaria total de cada nutriente se obtuvo sumando los aportes provenientes de todos los alimentos. En la tabla de Mataix Verdú hay muchos alimentos que son de características similares a los consumidos en Canarias como principios básicos, como la carne de conejo, el pollo, las asaduras, el gofio; ahora bien, hay alimentos que forman parte de los hábitos de consumo canarios como el potaje, el puchero, las papas con carne, la pastelería casera (el bizcochón, el flan o el quesillo) que no están en dicha tabla. En estos casos se procedió a obtener diversas recetas del guiso o postre y aplicar la más habitual y sencilla, procediéndose a la ejecución del mismo mediante pesada, teniendo en cuenta la porción comestible y las pérdidas nutricionales con la cocción de aquellos alimentos afectados. Posteriormente se sirvió una ración estándar y se pesó para reajustar los cálculos nutricionales.

Validación. Las Islas Canarias disfrutan de un clima subtropical con una media anual de temperatura de unos $20^{\circ} \mathrm{C}$, con discretas variaciones entre estaciones, lo que hace que, a excepción de la fruta de temporada, la alimentación del canario no cambie de forma sustancial entre el invierno y el verano.
Esperando obtener una muestra de 1.000 sujetos, se citó en los centros de salud, entre enero de 2000 y diciembre de 2005, a 1.200 personas de la población canaria entre 19 y 30 años, seleccionadas al azar de las tarjetas sanitarias, que cubren al $99 \%$ de la población de las islas que componen el archipiélago. Este rango de edad se elige para compararla con otra muestra de estudiantes universitarios con esas edades. A cada participante se le administró el cuestionario CDC-FFQ. El cuestionario fue administrado por encuestadores previamente entrenados. CDC-FFQ fue también administrado a 120 estudiantes de la Universidad de La Laguna, en los meses de octubre y noviembre del periodo 2004-2007. A estos estudiantes se les sometió a tres cuestionarios de tipo recordatorio de consumo de alimentos de 24 horas, el primero una semana después del CDC-FFQ. El tiempo entre recordatorios se intentó que no fuese superior a 7 días para evitar pérdidas. El periodo octubre-noviembre se eligió por tratarse de la época probablemente más templada y corresponder a fechas alejadas de los exámenes, para procurar aumentar así la participación.

Análisis de los datos. La muestra reunida se describe de manera resumida con porcentajes para las variables nominales, y media y desviación para las cuantitativas con distribución normal.

La validez del CDC-FFQ se estima a través de la comparación de nutrientes estimada por el cuestionario entre estudiantes universitarios y sujetos con nivel universitario de estudios en población general y entre estudiantes universitarios y población general en ese grupo de edad con cualquier nivel de estudios, empleando la prueba t-Student para muestras no relacionadas.

Se compara el consumo medio diario de nutrientes ajustado por la ingesta diaria de calorías entre el CDC-FFQ y el promedio de los tres recordatorios de 24 horas para los estudiantes universitarios. 
Se estiman las correlaciones entre nutrientes, brutas y ajustadas por la ingesta diaria de calorías, entre el cuestionario CDC-FFQ y el promedio de los tres recordatorios de 24 horas, en los estudiantes universitarios. Posteriormente se estiman las correlaciones entre grupos de alimentos según el cuestionario CDCFFQ y el promedio de los tres recordatorios de 24 horas, en los estudiantes universitarios. En estas estimaciones se utiliza el coeficiente lineal de Pearson o de Spearman, según la distribución de nutrientes y grupos de alimentos sea normal o no.
Se valora la concordancia de nutrientes ajustados por la ingesta media diaria de calorías totales consumidas del primer quintil del CDC-FFQ con el quinto quintil del promedio de los tres recordatorios de 24 horas y el quinto quintil del CDC-FFQ con el primer quintil del promedio de los tres recordatorios de 24 horas, para los estudiantes universitarios. Se obtienen las frecuencias de discordancia y el grado de acuerdo descartando la coincidencia por azar con el coeficiente kappa de Cohen. Un análisis similar se repite para los grupos de alimentos.

Tabla 1

Resultados del CDC-FFQ entre una muestra de estudiantes universitarios, una submuestra de población general con estudios universitarios y la muestra de población general de la misma edad y cualquier nivel de estudios

\begin{tabular}{|c|c|c|c|c|c|}
\hline \multirow[b]{2}{*}{ Consumo diario del nutriente* } & \multirow{2}{*}{$\begin{array}{c}\text { Muestra A: } \\
\text { Estudiantes universitarios } \\
19-30 \text { años } \\
(n=106)\end{array}$} & \multirow{2}{*}{$\begin{array}{c}\text { Muestra B: } \\
\text { Población general } \\
\text { de 19-30 años con } \\
\text { estudios universitarios } \\
(n=247)\end{array}$} & \multirow{2}{*}{$\begin{array}{c}\text { Muestra C: } \\
\text { Población general } \\
\text { de 19-30 años con } \\
\text { cualquier nivel } \\
\text { de estudios } \\
(n=1067)\end{array}$} & \multicolumn{2}{|c|}{ Significación } \\
\hline & & & & A-B & A-C \\
\hline Ingesta calórica (Kcal) & $2245 \pm 106,6$ & $2202,3 \pm 73,1$ & $2296,8 \pm 34,4$ & 0,744 & 0,652 \\
\hline Proteínas (g) & $103,9 \pm 4,9$ & $95,8 \pm 3,5$ & $94,7 \pm 1,5$ & 0,191 & 0,072 \\
\hline Grasas totales $(\mathrm{g})$ & $93,9 \pm 5,4$ & $88,8 \pm 3,7$ & $94,0 \pm 1,7$ & 0,448 & 0,985 \\
\hline Carbohidratos (g) & $251,2 \pm 13,6$ & $255,9 \pm 9,0$ & $271,6 \pm 4,3$ & 0,774 & 0,151 \\
\hline Grasas saturadas $(\mathrm{g})$ & $28,6 \pm 1,9$ & $26,3 \pm 1,3$ & $27,8 \pm 0,6$ & 0,317 & 0,686 \\
\hline Grasas monoinsaturadas $(\mathrm{g})$ & $33,1 \pm 1,8$ & $31,6 \pm 1,1$ & $34,3 \pm 0,56$ & 0,478 & 0,528 \\
\hline Grasa poliinsaturadas $(\mathrm{g})$ & $12,9 \pm 1,0$ & $12,3 \pm 0,4$ & $14,0 \pm 0,3$ & 0,476 & 0,252 \\
\hline Colesterol (mg) & $279,1 \pm 12,9$ & $263,2 \pm 8,5$ & $276,4 \pm 4,9$ & 0,283 & 0,867 \\
\hline Fibra (g) & $20,6 \pm 1,6$ & $19,3 \pm 0,8$ & $19,0 \pm 0,3$ & 0,476 & 0,362 \\
\hline Calcio (mg) & $1624,9 \pm 95,1$ & $1486,4 \pm 80,4$ & $1375,7 \pm 28,5$ & 0,315 & 0,009 \\
\hline Hierro (mg) & $35,3 \pm 3,0$ & $34,7 \pm 1,9$ & $32,7 \pm 1,0$ & 0,857 & 0,414 \\
\hline Magnesio (mg) & $379,5 \pm 21,2$ & $356,5 \pm 12,2$ & $354,4 \pm 5,4$ & 0,348 & 0,176 \\
\hline Sodio (mg) & $1584,6 \pm 94,5$ & $1559,0 \pm 56,9$ & $1696,1 \pm 30,2$ & 0,810 & 0,268 \\
\hline Potasio (mg) & $3794,3 \pm 218,7$ & $3568,3 \pm 119,8$ & $3563,7 \pm 55,7$ & 0,332 & 0,309 \\
\hline Fósforo (mg) & $1547,7 \pm 75,5$ & $1451,2 \pm 58,8$ & $1449,9 \pm 24,6$ & 0,348 & 0,232 \\
\hline Vitamina A (mg) & $1029,1 \pm 65,1$ & $946,5 \pm 40,0$ & $968,3 \pm 21,3$ & 0,270 & 0,395 \\
\hline Vitamina B1 (mg) & $1,8 \pm 0,1$ & $1,7 \pm 0,1$ & $1,6 \pm 0,01$ & 0,204 & 0,036 \\
\hline Vitamina B2 (mg) & $2,5 \pm 0,1$ & $2,3 \pm 0,1$ & $2,2 \pm 0,01$ & 0,263 & 0,009 \\
\hline Vitamina B6 (mg) & $3,5 \pm 0,4$ & $3,3 \pm 0,4$ & $3,0 \pm 0,1$ & 0,794 & 0,302 \\
\hline Vitamina B12 (mg) & $11,8 \pm 0,8$ & $9,3 \pm 0,3$ & $9,4 \pm 0,2$ & 0,004 & 0,004 \\
\hline Niacina (mg) & $31,5 \pm 1,5$ & $28,8 \pm 1,1$ & $26,9 \pm 0,4$ & 0,153 & 0,002 \\
\hline Acido Fólico (mg) & $338,8 \pm 21,0$ & $316,4 \pm 12,2$ & $298,1 \pm 5,0$ & 0,335 & 0,062 \\
\hline Vitamina C (mg) & $282,5 \pm 26,2$ & $296,2 \pm 12,9$ & $269,5 \pm 5,8$ & 0,602 & 0,628 \\
\hline Vitamina D (mg) & $7,6 \pm 0,6$ & $6,2 \pm 0,3$ & $5,7 \pm 0,1$ & 0,052 & 0,005 \\
\hline Vitamina E (mg) & $12,1 \pm 0,8$ & $11,4 \pm 0,4$ & $11,8 \pm 0,2$ & 0,362 & 0,715 \\
\hline
\end{tabular}

* Media \pm Error Estándar de la Media. 
Todos los cálculos fueron realizados con el programa SPSS versión 15.0 y la significación estadística para todas las pruebas empleadas fue establecida al nivel bilateral en 0,05 .

\section{RESULTADOS}

La muestra final de población general canaria quedó conformada por 1.067 sujetos de 19 a 30 años, de los cuales el $57 \%$ eran mujeres. 247 personas de esta muestra tenían estudios universitarios, y de ellos el $65 \%$ eran mujeres. La distribución de la muestra según islas fue la siguiente: $38 \%$ de Tenerife, $37 \%$ de Gran Canaria, 9\% de Lanzarote, $6 \%$ de La Gomera, 5\% de Fuerteventura y 5\% de La Palma. En cuanto a la muestra de estudiantes universitarios, quedó conformada por 106 sujetos, de los que el $59 \%$ eran mujeres.

En la tabla 1 se muestran los resultados de la comparación de nutrientes obtenidos con el cuestionario CDC-FFQ entre los estudiantes universitarios y entre los sujetos con estudios universitarios de la población general, y entre los estudiantes universitarios y la población general de 19 a 30 años.

En la tabla 2 se presentan los resultados de la comparación de la ingesta media dia-

Tabla 2

Comparación del consumo medio diario de nutrientes ajustado por la ingesta calórica total, según es estimado por el cuestionario CDC-FFQ y el recordatorio de 24 horas

(promedio de tres administraciones consecutivas) en una muestra de 106 estudiantes universitarios

\begin{tabular}{|c|c|c|c|}
\hline Nutriente $^{1}$ & CDC-FFQ $^{2}$ & Recordatorio & Significación $^{3}$ \\
\hline Kcal/día & $2245 \pm 106,6$ & $1538 \pm 388,9$ & $<0,001$ \\
\hline Proteínas (g/Kcal día) & $46,7 \pm 11,1$ & $53,3 \pm 13,6$ & $<0,001$ \\
\hline Grasas totales (g/Kcal día) & $41,0 \pm 9,5$ & $35,3 \pm 8,5$ & $<0,001$ \\
\hline Carbohidratos (g/Kcal día) & $113,3 \pm 26,6$ & $114,5 \pm 26,6$ & 0,649 \\
\hline Grasas saturadas (g/Kcal día) & $12,4 \pm 4,4$ & $12,4 \pm 3,6$ & 0,988 \\
\hline Grasas monoinsaturadas (g/Kcal día) & $14,8 \pm 4,4$ & $11,9 \pm 4,8$ & $<0,001$ \\
\hline Grasa poliinsaturadas (g/Kcal día) & $5,7 \pm 2,2$ & $3,6 \pm 1,8$ & $<0,001$ \\
\hline Colesterol (mg/Kcal día) & $129,2 \pm 45,5$ & $182,0 \pm 68,1$ & $<0,001$ \\
\hline Fibra (g/Kcal día) & $8,9 \pm 3,7$ & $10,1 \pm 4,3$ & 0,003 \\
\hline Calcio (mg/Kcal día) & $718,6 \pm 241,5$ & $666,9 \pm 235,5$ & 0,041 \\
\hline Hierro (mg/Kcal día) & $16,5 \pm 3,7$ & $11,4 \pm 4,4$ & 0,001 \\
\hline Magnesio (mg/Kcal día) & $170,8 \pm 48,1$ & $185,2 \pm 46,7$ & 0,001 \\
\hline Sodio (mg/Kcal día) & $711,2 \pm 248,9$ & $766,4 \pm 278,2$ & 0,093 \\
\hline Potasio (mg/Kcal día) & $1,706,8 \pm 520,4$ & $1,859,6 \pm 425,2$ & 0,004 \\
\hline Fósforo (mg/Kcal día) & $689,8 \pm 139,3$ & $782,9 \pm 183,5$ & $<0,001$ \\
\hline Vitamina A (mg/Kcal día) & $455,5 \pm 191,9$ & $460,8 \pm 241,7$ & 0,825 \\
\hline Vitamina B1 ( $\mu \mathrm{g} /$ Kcal día) & $0,8 \pm 0,2$ & $0,9 \pm 0,4$ & 0,282 \\
\hline Vitamina B2 ( $\mu \mathrm{g} /$ Kcal día) & $1,1 \pm 0,4$ & $1,2 \pm 0,4$ & 0,097 \\
\hline Vitamina B6 ( $\mu \mathrm{g} /$ Kcal día) & $1,6 \pm 0,3$ & $1,5 \pm 0,4$ & 0,281 \\
\hline Vitamina B12 ( $\mu \mathrm{g} /$ Kcal día $)$ & $5,2 \pm 2,4$ & $5,1 \pm 2,8$ & 0,754 \\
\hline Niacina ( $\mu \mathrm{g} /$ Kcal día) & $14,5 \pm 4,8$ & $16,4 \pm 5,0$ & 0,002 \\
\hline Acido Fólico ( $\mu \mathrm{g} / \mathrm{Kcal}$ día) & $151,4 \pm 54,3$ & $151,9 \pm 50,9$ & 0,919 \\
\hline Vitamina C ( $\mu \mathrm{g} / \mathrm{Kcal}$ día $)$ & $126,3 \pm 79,9$ & $132,7 \pm 77,4$ & 0,350 \\
\hline Vitamina D ( $\mu \mathrm{g} /$ Kcal día $)$ & $3,5 \pm 0,6$ & $3,1 \pm 0,8$ & 0,341 \\
\hline Vitamina E (mg/Kcal día) & $5,3 \pm 1,7$ & $4,5 \pm 1,7$ & $<0,001$ \\
\hline
\end{tabular}

1 Ajustado al consumo medio diario energético por cada $1.000 \mathrm{Kcal}$.

2 Media \pm Desviación típica.

3 Prueba t-Student para muestras relacionadas. 
Tabla 3

Correlación de nutrientes entre CDC-FFQ

y los valores promedio de tres recordatorios de 24 horas en una muestra de 106 estudiantes universitarios

\begin{tabular}{|c|c|c|c|c|}
\hline \multirow{3}{*}{$\begin{array}{c}\begin{array}{c}\text { Consumo diario } \\
\text { del nutriente }\end{array} \\
\text { Ingesta calórica (Kcal) }\end{array}$} & \multicolumn{4}{|c|}{ Correlación* } \\
\hline & \multicolumn{2}{|c|}{$\begin{array}{l}\text { No ajustada } \\
\text { significación }\end{array}$} & \multicolumn{2}{|c|}{$\begin{array}{l}\text { Ajustadas } \\
\text { significación }\end{array}$} \\
\hline & 0,290 & 0,003 & - & - \\
\hline Proteínas (g) & 0,273 & 0,005 & 0,248 & 0,010 \\
\hline Grasas totales (g) & 0,328 & 0,001 & 0,468 & $<0,001$ \\
\hline Carbohidratos (g) & 0,348 & $<0,001$ & 0,426 & $<0,001$ \\
\hline Grasas saturadas (g) & 0,377 & $<0,001$ & 0,405 & $<0,001$ \\
\hline Grasas monoinsaturadas (g) & 0,287 & 0,002 & 0,394 & $<0,001$ \\
\hline Grasa poliinsaturadas (g) & 0,397 & $<0,001$ & 0,401 & $<0,001$ \\
\hline Colesterol (mg) & 0,286 & 0,003 & 0,292 & 0,002 \\
\hline Fibra (g) & 0,491 & $<0,001$ & 0,463 & $<0,001$ \\
\hline Calcio (mg) & 0,239 & 0,014 & 0,419 & $<0,001$ \\
\hline Hierro (mg) & 0,202 & 0,038 & 0,272 & 0,005 \\
\hline Magnesio (mg) & 0,316 & 0,001 & 0,581 & $<0,001$ \\
\hline Sodio $(\mathrm{mg})$ & 0,343 & $<0,001$ & 0,214 & 0,028 \\
\hline Potasio (mg) & 0,455 & $<0,001$ & 0,392 & $<0,001$ \\
\hline Fósforo (mg) & 0,328 & 0,001 & 0,331 & 0,001 \\
\hline Vitamina A $(\mu \mathrm{g})$ & 0,395 & $<0,001$ & 0,376 & $<0,001$ \\
\hline Vitamina B1 (mg) & 0,173 & 0,076 & 0,103 & 0,297 \\
\hline Vitamina B2 (mg) & 0,250 & 0,010 & 0,427 & $<0,001$ \\
\hline Vitamina B6 (mg) & 0,225 & 0,020 & 0,278 & 0,004 \\
\hline Vitamina B12 $(\mu \mathrm{g})$ & 0,228 & 0,019 & 0,202 & 0,036 \\
\hline Niacina (mg) & 0,267 & 0,006 & 0,297 & 0,002 \\
\hline Acido Fólico $(\mu \mathrm{g})$ & 0,447 & $<0,001$ & 0,537 & $<0,001$ \\
\hline Vitamina C (mg) & 0,595 & $<0,001$ & 0,601 & $<0,001$ \\
\hline Vitamina D $(\mu \mathrm{g})$ & 0,361 & $<0,001$ & 0,366 & $<0,001$ \\
\hline Vitamina E (mg) & 0,395 & $<0,001$ & 0,397 & $<0,001$ \\
\hline
\end{tabular}

* Coeficiente de correlación de Spearman o de Pearson según distribución de la variable.

No ajustado: correlación empleando la estimación bruta de la ingesta. Ajustado: correlación empleando la estimación de ingesta ajustada al consumo de calorías diarias.

ria de nutrientes ajustada por el consumo diario de cada mil Kilocalorías entre el CDC-FFQ y el promedio de los tres recordatorios de 24 horas para los estudiantes universitarios.

En la tabla 3 se exponen las correlaciones entre los nutrientes estimados con el CDC-FFQ y los obtenidos de la media de los tres recordatorios de 24 horas, para la muestra de estudiantes universitarios, con las estimaciones de consumo en bruto $\mathrm{y}$ ajustadas por el consumo diario de calorías.
Tabla 4

Correlación entre la ingesta diaria estimada para los grupos de alimentos entre el CDC-FFQ y la media de tres recordatorios de $\mathbf{2 4}$ horas en una muestra de 106 estudiantes universitarios

\begin{tabular}{|l|c|c|}
\hline $\begin{array}{c}\text { Ingesta diaria según grupos } \\
\text { de alimentos (gr/día) }\end{array}$ & Correlación* & Significación \\
\hline Cereales & 0,450 & $<0,001$ \\
\hline Lácteos & 0,371 & $<0,001$ \\
\hline Dulces & 0,345 & $<0,001$ \\
\hline Féculas & 0,205 & 0,034 \\
\hline Azúcares & 0,434 & $<0,001$ \\
\hline Frutas & 0,543 & $<0,001$ \\
\hline Carnes & 0,381 & $<0,001$ \\
\hline Huevos & 0,289 & 0,003 \\
\hline Embutidos & 0,299 & 0,002 \\
\hline Pescados & 0,280 & 0,004 \\
\hline Verduras & 0,542 & $<0,001$ \\
\hline Frutos secos & 0,248 & 0,010 \\
\hline Bebidas alcohólicas & 0,243 & 0,012 \\
\hline Café e infusiones & 0,148 & 0,129 \\
\hline Refrescos & 0,413 & $<0,001$ \\
\hline Agua & 0,339 & $<0,001$ \\
\hline Otras bebidas no alcohólicas & 0,319 & 0,001 \\
\hline
\end{tabular}

* Coeficiente de correlación de Spearman o Pearson según distribución de la variable.

La vitamina B1 ajustada al consumo calórico no presentó correlación entre los dos métodos de estimación $(p=0,297)$, mientras que el resto de los nutrientes presentaron correlaciones que oscilaron entre 0,202 y 0,601 .

Las correlaciones para la muestra de estudiantes universitarios entre los grupos de alimentos estimados con el CDC-FFQ y los obtenidos de la media de los tres Recordatorios de 24 horas se muestran en la tabla 4. En este caso, no hubo correlación en el grupo de café e infusiones $(\mathrm{p}=0,129)$, mientras que en los restantes grupos las correlaciones oscilaron entre 0,205 y 0,543 .

En la tabla 5 se presenta el desacuerdo en el consumo medio de nutrientes según se estima por el CDC-FFQ y el promedio de los tres recordatorios de 24 horas, para los estudiantes universitarios, mediante la cla- 
Tabla 5

Desacuerdo en la estimación del consumo medio diario de nutrientes ajustados por la ingesta de calorías según el cuestionario CDC-FFQ y el promedio de tres recordatorios de 24 horas en una muestra de 106 estudiantes universitarios. Clasificación cruzada en los quintiles extremos y concordancia global de la clasificación del consumo

\begin{tabular}{|c|c|c|c|c|}
\hline \multirow{5}{*}{$\begin{array}{c}\text { Consumo medio } \\
\text { diario del nutriente }\end{array}$} & \multirow{2}{*}{\multicolumn{2}{|c|}{\begin{tabular}{|c|}
$\begin{array}{c}\text { Desacuerdo } \\
\text { (valores absolutos) }\end{array}$ \\
CDC-FFQ Quintil \\
\end{tabular}}} & \multirow{5}{*}{$\begin{array}{c}\text { Concordancia }{ }^{2} \\
(\%)\end{array}$} & \multirow{5}{*}{$\begin{array}{c}\text { Significación } \\
\text { de la } \\
\text { concordancia }\end{array}$} \\
\hline & & & & \\
\hline & Más bajo & Más alto & & \\
\hline & \multicolumn{2}{|c|}{ R24h ${ }^{1}$ Quintil } & & \\
\hline & Más alto & Más bajo & & \\
\hline Proteínas & 1 & 2 & 61,3 & 0,013 \\
\hline Grasas totales & 3 & 2 & 45,1 & 0,048 \\
\hline Carbohidratos & 1 & 2 & 65,8 & 0,005 \\
\hline Grasas saturadas & 2 & 1 & 71,5 & 0,001 \\
\hline $\begin{array}{l}\text { Grasas } \\
\text { monoinsaturadas }\end{array}$ & 1 & 2 & 62,6 & 0,009 \\
\hline $\begin{array}{l}\text { Grasas } \\
\text { poliinsaturadas }\end{array}$ & 2 & 3 & 45,7 & 0,043 \\
\hline Colesterol & 1 & 2 & 62,8 & 0,009 \\
\hline Fibra & 1 & 0 & 90,4 & $<0,001$ \\
\hline Calcio & 2 & 3 & 61,5 & 0,002 \\
\hline Hierro & 4 & 3 & 22,2 & 0,343 \\
\hline Magnesio & 2 & 0 & 83,3 & $<0,001$ \\
\hline Sodio & 6 & 1 & 39,4 & 0,040 \\
\hline Potasio & 0 & 0 & 100 & $<0,001$ \\
\hline Fósforo & 3 & 3 & 47,7 & 0,022 \\
\hline Vitamina A & 0 & 0 & 100 & $<0,001$ \\
\hline Vitamina B1 & 6 & 2 & 22,3 & 0,279 \\
\hline Vitamina B2 & 1 & 2 & 70,0 & 0,002 \\
\hline Vitamina B6 & 1 & 3 & 58,8 & 0,007 \\
\hline Vitamina B12 & 3 & 3 & 29,2 & 0,229 \\
\hline Niacina & 3 & 0 & 72,0 & 0,001 \\
\hline Acido Fólico & 0 & 1 & 91,7 & $<0,001$ \\
\hline Vitamina C & 0 & 1 & 91,3 & $<0,001$ \\
\hline Vitamina D & 1 & 2 & 61,3 & 0,013 \\
\hline Vitamina E & 1 & 1 & 81,7 & $<0,001$ \\
\hline
\end{tabular}

1-Valor medio de los tres recordatorios de 24 horas. 2-Según el estadístico Kappa de Cohen.

sificación de sujetos en los quintiles extremos opuestos de cada medición y la concordancia global para cada nutriente. No presentaron concordancia el hierro $(\mathrm{p}=0,343)$ y las vitaminas B1 $\quad(\mathrm{p}=0,279)$ y $\quad \mathrm{B} 12$ $(\mathrm{p}=0,229)$. El resto de nutrientes presentó concordancias que oscilaron desde el 45,1 al $100 \%$.
Tabla 6

Desacuerdo en la estimación del consumo medio diario de grupos de alimentos según el cuestionario CDC-FFQ y el promedio de tres recordatorios de $\mathbf{2 4}$ horas en una muestra de 106 estudiantes universitarios.

Clasificación cruzada en los quintiles extremos y concordancia global de la clasificación del consumo

\begin{tabular}{|c|c|c|c|c|}
\hline \multirow{5}{*}{$\begin{array}{l}\text { Consumo medio } \\
\text { diario del nutriente }\end{array}$} & \multirow{2}{*}{\multicolumn{2}{|c|}{\begin{tabular}{|c} 
Desacuerdo \\
(valores absolutos)
\end{tabular}}} & \multirow{5}{*}{$\begin{array}{c}\text { Concordancia } \\
(\%)\end{array}$} & \multirow{5}{*}{$\begin{array}{c}\text { Significación } \\
\text { de la } \\
\text { concordancia }\end{array}$} \\
\hline & & & & \\
\hline & Más bajo & Más alto & & \\
\hline & \multicolumn{2}{|c|}{ R24h ${ }^{1}$ Quintil } & & \\
\hline & Más alto & Más bajo & & \\
\hline Cereales & 0 & 1 & 85,1 & 0,001 \\
\hline Lácteos & 2 & 3 & 50,0 & 0,025 \\
\hline Dulces & 3 & 1 & 61,5 & 0,004 \\
\hline Féculas & 0 & 7 & 42,1 & 0,015 \\
\hline Azúcares & 0 & 0 & 100 & $<0,001$ \\
\hline Frutas & 1 & 1 & 82,6 & $<0,001$ \\
\hline Carnes & 0 & 0 & 100 & $<0,001$ \\
\hline Huevos & 3 & 3 & 41,0 & 0,038 \\
\hline Embutidos & 3 & 3 & 43,5 & 0,023 \\
\hline Pescados & 2 & 2 & 60,7 & 0,004 \\
\hline Verduras & 1 & 3 & 67,7 & $<0,001$ \\
\hline Frutos secos & 0 & 0 & 100 & $<0,001$ \\
\hline $\begin{array}{l}\text { Bebidas } \\
\text { alcohólicas }\end{array}$ & 0 & 0 & 100 & $<0,001$ \\
\hline $\begin{array}{l}\text { Café e } \\
\text { infusiones }\end{array}$ & 3 & 2 & 35,5 & 0,152 \\
\hline Refrescos & 0 & 0 & 100 & $<0,001$ \\
\hline Agua & 1 & 1 & 75,7 & 0,002 \\
\hline $\begin{array}{l}\text { Otras bebidas } \\
\text { no alcohólicas }\end{array}$ & 0 & 0 & 100 & $<0,001$ \\
\hline
\end{tabular}

1 Valor medio de los tres recordatorios de 24 horas. 2 Según el estadístico Kappa de Cohen.

En la tabla 6 se expone el desacuerdo en el consumo medio de grupos de alimentos según se estima por el CDC-FFQ y el promedio de los tres recordatorios de 24 horas, para los estudiantes universitarios, mediante la clasificación de sujetos en los quintiles extremos opuestos de cada medición y la concordancia global para cada grupo de alimentos. Las concordancias en este caso quedaron comprendidas entre el $41 \%$ y el $100 \%$ para todos los grupos excepto para el de café e infusiones donde dicha concordancia no se observó ( $\mathrm{p}=0,152)$. 


\section{DISCUSIÓN}

En nuestro conocimiento el CDC-FFQ es el primer cuestionario para el que se publica su validación respecto de la ingesta de alimentos y nutrientes en la población canaria. El CDC-FFQ es una versión reformada y ampliada del cuestionario de Martin Moreno JM y cols. ${ }^{7}$ adaptada a las peculiaridades de la dieta canaria, al que se le han añadido alimentos típicos de la región y modificado la forma de estimar la cantidad y frecuencia de su consumo.

Consideramos que las pruebas realizadas sobre el CDC-FFQ muestran que este cuestionario posee la suficiente validez para medir la ingesta media diaria de grupos de alimentos y nutrientes. La validez del CDC-FFQ es sólo aplicable a la población en la que se ha probado, canarios adultos jóvenes entre 19 y 30 años. Aunque los recordatorios empleados en su validación corresponden a población universitaria, puede deducirse la utilidad de CDC-FFQ para la población general, pues no se observa diferencia en el consumo de nutrientes, excepto para el calcio y algunas vitaminas, lo que podría deberse a una diferencia en la cantidad o calidad consumida más que a un defecto de medición. Por otro lado, a la población general se le administró el cuestionario CDC-FFQ mediante entrevista y para los estudiantes universitarios fue autocumplimentado. Creemos que el nivel cultural de los estudiantes universitarios minimiza el sesgo de imprecisión del cuestionario autoadministrado, lo que se aprecia en la no diferencia de sus resultados con los de la población general con estudios universitarios. Algún otro cuestionario de alimentación, aunque más breve, se ha mostrado posteriormente válido en adultos jóvenes y en personas de mayor edad. ${ }^{11}$

Resulta llamativa la baja correlación del consumo de frutos secos y bebidas alcohólicas entre el cuestionario CDC-FFQ y el promedio de los tres recordatorios de 24 horas, con la alta concordancia que se observa en estas declaraciones de ingesta entre sus quintiles extremos. La explicación del fenómeno podría hallarse en una baja correlación en los intervalos situados entre los quintiles extremos y una alta correlación en los extremos mismos. Esta sospecha no se pudo comprobar debido a la imposibilidad de estimar la correlación en la escasa submuestra que cumple la condición de encontrarse en el mayor quintil de consumo de alcohol y frutos secos tanto en el CDC-FFQ como en los recordatorios de 24 horas. Esta escasez de muestra se explica porque se consume más alcohol, muchas veces acompañada su ingesta por frutos secos, los fines de semana y en los periodos vacacionales, y los recordatorios de 24 horas se realizaron en octubre-noviembre y no exploraron el consumo de viernes y sábados.

Una de las limitaciones de este trabajo ha sido la imposibilidad de valorar la reproducibilidad del CDC-FFQ mediante la replicación de su administración a una misma muestra y la estimación de la correlación de las respuestas. La intención inicial era obtener cuatro recordatorios de 24 horas y dos CDC-FFQ en 120 estudiantes, pero el bajo nivel de respuesta en la cuarta administración del recordatorio de 24 horas y segunda del CDC-FFQ imposibilitó disponer de estos resultados. Una alternativa para estimar la reproducibilidad podría haber sido la estrategia de partición del CDC-FFQ en dos mitades con ítems correspondientes a grupos de alimentos similares en cada uno, valorando después la correlación entre ambas mitades, pero este enfoque no nos pareció acertado, debido a la subjetividad inherente a la repartición de ítems.

Es cierto que otros estudios utilizan registros de 3 a 7 días que miden la ingesta actual, teniendo en cuenta todos los alimentos que se consumen dentro y fuera de casa. Este es un método preciso si existe 
una buena colaboración ya que requiere un gran esfuerzo por parte de los participantes del estudio. Nosotros empleamos el recordatorio de 24 horas porque es rápido y relativamente sencillo de realizar, requiere una colaboración mínima, es aplicable a la mayoría de los grupos de la población, y el coste es bajo. Se sabe que dentro de las principales fuentes de error en los estudio de consumo alimentario existe la tendencia a la sobrestimación de las ingestas pequeñas o se subestiman las ingestas elevadas en los métodos de recuerdo. Es probable que algo de esto pueda explicar la diferencia para el consumo energético global y de algunos nutrientes entre el FFQ administrado a los estudiantes y la media de los tres recordatorios de 24 horas. Pensamos que esto obedece al hecho de que el CDC-FFQ estima el estilo global de alimentación al establecer una valoración de la dieta en general, en el último año, mientras los recordatorios de 24 horas son valoraciones puntuales de la dieta del día anterior al que se realiza. Además, en nuestro caso, los recordatorios de 24 horas, como hemos comentado, no valoraron el consumo de alimentos los viernes y sábados, días de la semana en que probablemente los estudiantes modifican más sus hábitos alimenticios semanales. La infra-declaración en la ingesta de calorías en los recordatorios de 24 horas respecto a los cuestionarios de frecuencia de consumo ha sido apreciada en otros estudios de valoración del consumo de alimentos aplicados en población hispana ${ }^{12-13}$. Entre los factores que se han señalado como posibles causas de esta infra-declaración en los recordatorios de 24 horas se encuentran la insatisfacción con el propio cuerpo y el seguir una dieta para perder peso durante el último año ${ }^{14}$. El que la prueba del CDC-FFQ se haya realizado en adultos jóvenes estudiantes universitarios, personas mayoritariamente preocupadas por su aspecto físico, podría haber influido en alguna medida en una infra-declaración del consumo global de alimentos.
Acorde a los resultados de la validación, y teniendo en cuenta las características que debe reunir un cuestionario de frecuencia de consumo de alimentos ${ }^{15}$, consideramos que el CDC-FFQ es un instrumento que muestra buenas propiedades de consistencia externa, lo que lo convierte en un medio capaz de clasificar razonablemente a los sujetos de 19 a 30 años de la población canaria en los rangos relativos de su nivel de ingesta de alimentos y nutrientes, y que por ello podría resultar de utilidad en estudios epidemiológicos donde se necesite valorar la dieta de este grupo de población en las Islas Canarias.

\section{BIBLIOGRAFÍA}

1. Martín-Moreno JM, Gorgojo L. Valoración de la ingesta dietética a nivel poblacional mediante cuestionarios individuales: sombras y luces metodológicas. Rev Esp Salud Publica 2007; 81: 507518.

2. Willet WC. Nutritional Epidemiology. 2nd ed. Oxford: Oxford University Press; 1998.

3. Block G, Hartman AM, Dresser CM, Carroll MD, Gannon J, Gardner L. A data based approach to diet questionnaire design and testing. Am J Epidemiol 1986; 24: 453-69.

4. Margetts BM, Nelson M. Design Concepts in Nutrition Epidemiology. Oxford: Oxford University Press; 1997.

5. Benito E, Stiggelbout A, Bosch FX, Obrador A, Kaldor J, Mulet M, Muñoz N. Nutritional factors in colorectal cancer risk: a case-control study in Majorca. Int J Cancer 1991; 49: 161-167.

6. Riboli E, González CA, López-Abente G, Errezola M, Izarzugaza I, Escolar A, Nebot M, Hemon B, Agudo A. Diet and bladder cancer in Spain: a multi-centre case control study. Int J Cancer 1991; 49: 214-219.

7. Martín Moreno JM, Boyle P, Gorgojo L, Mainsonneuve $\mathrm{P}$, Fernández-Rodríguez JC, Salvini S, Willet WC. Development and validation of a food frequency questionnaire in Spain. Int J Epidemiol 1993; 22: 512-9.

8. Encuesta nutricional de Canarias 1997-1998. Gobierno de Canarias. Disponible en: www.gobier- 
nodecanarias.org/sanidad/scs/1/plansalud/enca/tom ol/index.htm

9. Cabrera de León A, Rodríguez Pérez MC, del Castillo Rodríguez JC, Brito Díaz B, Pérez Méndez LI, Muros de Fuentes M, Almeida González D, Batista Medina M, Aguirre-Jaime A. Estimación del riesgo coronario de la población canaria aplicando la ecuación de Framingham. Med Clín (Barc) 2006; 126: 521-6.

10. Mataix J, Mañas M, Llopis J, Martínez de Vitoria E. Tabla de composición de alimentos españoles. $4^{a}$ ed. Granada: Universidad de Granada; 1998.

11. Mochari H, Gao Q, Mosca L J. Validation of the MEDFICTS dietary assessment questionnaire in a diverse population. Am Diet Assoc. 2008; 108: 817-22.

12. Serra Majem L, Morales D, Domingo C, Caubet E, Ribas L, Nogués RM. Comparación de dos métodos de valoración de la ingesta de alimentos y nutrientes: recordatorio de 24 horas y cuestionario de frecuencia semicuantitativo. Med Clin (Barc) 1994; 103: 652-6.

13. Block G, Wakimoto P, Jensen C, Mandel S, Green RR. Validation of a food frequency questionnaire for Hispanics. Prev Chronic Dis 2006; 3: A77.

14. Novotny JA, Rumpler WV, Riddick H, Hebert JR, Rhodes D, Judd JT, Baer DJ, McDowell M, Briefel R. Personality characteristics as predictors of underreporting of energy intake on 24-hour dietary recall interviews. J Am Diet Assoc 2003 ; 103: 1146-51.

15. Cade J, Thompson, Burley V, Warm D. Development, validation and utilisation of food-frequency questionnaires- a review. Public Health Nutrition 2002; 5: 567-587. 